\title{
Computational Study of Polarizabilities and Second Hyperpolarizabilities of Inorganic Transition Metal Thiometalates and Metalates in Solution ${ }^{\dagger}$
}

\author{
Thomas R. Cundari, ${ }^{*, 1}$ Henry A. Kurtz, and Tie Zhou \\ Department of Chemistry, Computational Research on Materials Institute (CROMIUM), University of Memphis, \\ Memphis, Tennessee 38152-6060
}

Received: October 28, 1999; In Final Form: December 21, 1999

\begin{abstract}
A systematic study of nonlinear optical (NLO) properties of inorganic transition metal (TM) thiometalates and metalates is reported. Polarizabilities $(\alpha)$ and second hyperpolarizabilities $(\gamma)$ are calculated in solution within the polarizable continuum model. It is found that NLO properties of anionic inorganic complexes can be successfully modeled in solution, when this cannot be done so in the gas phase. Solvent effects are found to significantly increase $\alpha$ and $\gamma$. The effects are stronger on $\gamma$ (up to 80\%) than on $\alpha$ (up to 40\%) and stronger on TM thiometalates than on metalates. For $\alpha$, solvent effects are found to be more important than electron correlation effects. For $\gamma$, the two effects are similarly important. Solvent effects on $\alpha$ and $\gamma$ caused by subordinate factors other than the dominant electrostatic solute-solvent interactions were studied and assessed to be negligible. Upon solvation, large TM and ligand modification effects on $\alpha$ and $\gamma$ are found. One oxo-to-sulfido substitution results in an increase in $\alpha$ by 38 au and $\gamma$ by $10000 \mathrm{au}$.
\end{abstract}

\section{Introduction}

With the progress in computational modeling of nonlinear optical (NLO) properties, many important effects (e.g., basis set, electron correlation, solvent, intermolecular interaction, etc.) regarding NLO properties have been studied separately by different researchers mainly focusing on specific and/or prototypical small organic and organometallic molecules. ${ }^{2}$ However, systematic studies of NLO properties of inorganic complexes are comparatively very rare. ${ }^{3}$ As an initial effort to fill this void, we have recently studied NLO properties of all the existing transition metal $(\mathrm{TM})$ metalates, $\left[\mathrm{MO}_{4}\right]^{q-}(\mathrm{M}$ : transition metals of groups 4-8 and the first to third transition series, except for $\mathrm{Fe} ; q$ : charge). ${ }^{4}$ Metalates are simple molecular models for inorganic NLO materials, such as lithium niobate and barium titanate, ${ }^{5}$ which belong to the first NLO materials in use. To our knowledge, no experimental studies of the NLO properties of thiometalate species have been reported. Computations on organic systems suggest that oxygen $\rightarrow$ sulfur replacements can enhance NLO properties. ${ }^{6}$ For this reason, we undertook an evaluation of oxygen $\rightarrow$ sulfur replacements on NLO properties of inorganic species. Systematic research focusing on families of chemically and structurally correlated complexes contributes to understanding the relationship between NLO properties and chemical structure and properties, which is important for the design of novel NLO materials.

On the basis of the initial results for $\left[\mathrm{MO}_{4}\right]^{q-},{ }^{4 a}$ we found that NLO properties of neutral and sometimes less negatively charged inorganic species can be reliably modeled in the gas phase. However, there is a systematic overestimation of polarizabilities $(\alpha)$ and second hyperpolarizabilities $(\gamma)$ for highly negatively charged species as a consequence of the inherent difficulties in treating such species quantum mechanically in the gas phase. It is often the case that their valence electrons

\footnotetext{
$\dagger$ Part of the special issue "Electronic and Nonlinear Optical Materials Theory and Modeling".

* Address correspondence to this author.
}

are not bound to atomic or molecular orbitals, and thus they are not stable, gas-phase entities. Therefore, quantum calculations on isolated highly negatively charged species in a vacuum using extended basis sets, including diffuse polarization functions, give rise to magnifications in $\alpha$ and particularly in $\gamma{ }^{7}$ These are highly dependent on the electron density distribution in the outer and fringe region. The supermolecule approach with ligation of counterions (e.g., alkali metal cations) has proven to be successful in solving problems with modeling the NLO properties of anionic inorganic complexes. ${ }^{4 b}$

Another way to solve this problem is prompted by our interest in modeling NLO properties of anionic species in a solution environment as well as studying the resulting solvent effects on NLO properties of inorganic TM complexes. This is of interest because a study of solvent effects on NLO properties helps fill in the gap between quantum mechanical calculations done mostly for isolated molecules and the experimental measurements performed mostly in condensed phase (solution, solid). The screening of electron-electron repulsions on the anionic solute by a high dielectric solvent results in bound molecular orbitals. The (hyper)polarizabilities of this stable form can then be reliably calculated by current techniques.

Thus, as a further step in a systematic study of the NLO properties of inorganic TM complexes, this present research was extended from TM metalates, $\left[\mathrm{MO}_{4}\right]^{q-}$, to all known TM thiometalates, $\left[\mathrm{MS}_{4}\right]^{q-}(\mathrm{M}$ : transition metals of groups 5-7 and the first to third transition series; $q$ : charge), and from the gas-phase isolated systems to solution. The research employed a sophisticated solvation model technique, the polarizable continuum model (PCM). ${ }^{8-12}$ Recently, some works on solvent effects on (hyper)polarizabilities for some prototypical organic molecules using the PCM technique have been reported. ${ }^{13-16}$ However, to our knowledge, this present work is the first systematic study of solvent effects on NLO properties of inorganic complexes. In addition, reliable modeling of NLO properties for a series of TM thiometalates versus metalates in 
solution made possible a study of another important effect, the ligand modification effect.

\section{Computational Methods}

Polarizabilities $(\alpha)$ and second hyperpolarizabilities $(\gamma)$ were calculated at the restricted Hartree-Fock (RHF) level using the time-dependent Hartree-Fock (TDHF) method. ${ }^{17}$ Correlated Møller-Plesset second-order perturbation theory (MP2) level calculations employed the finite field (FF) method. ${ }^{18}$ All calculations were done with the GAMESS ${ }^{19}$ program. The reported $\langle\alpha\rangle$ and $\langle\gamma\rangle$ results are the static field (DC) isotropic average values defined by

$$
\langle\alpha\rangle=\left(\alpha_{x x}+\alpha_{y y}+\alpha_{z z}\right) / 3
$$

and

$$
\langle\gamma\rangle=\left[\gamma_{x x x x}+\gamma_{y y y y}+\gamma_{z z z z}+2\left(\gamma_{x x y y}+\gamma_{x x z z}+\gamma_{y y z z}\right)\right] / 5
$$

The first hyperpolarizability $(\beta)$ is excluded from this study as it is zero by symmetry for most of the target species of interest.

Special GAMESS options regarding the SCF procedure for reliable FF and TDHF calculations were reported in previous papers. ${ }^{4}$ In addition, for FF calculations, a field strength of 0.005 au was applied (default, $0.001 \mathrm{au}$ ) to minimize the numerical problems associated with the FF procedure. ${ }^{2 \mathrm{~b}}$ This choice was based on a symmetry analysis of equivalent tensor parameters as well as a comparison with the TDHF results.

$\mathrm{PCM}^{8,9}$ for solvation has been extended to coupled perturbed Hartree-Fock (CPHF) theory and implemented in recent versions of GAMESS to calculate solute (hyper)polarizabilities at the RHF level. In PCM, the solvent is treated as a continuum distribution of a dielectric medium with the solute embedded in a cavity in the continuum medium. The shape of the cavity is defined in terms of interlocking spheres centered on the solute nuclei, with electric charges distributed on the surface. The size of the cavity is defined from the van der Waals radii $\left(R_{\mathrm{vdW}}\right)$ of solute atoms multiplied by a factor, the default of which is 1.2 in GAMESS, and was applied in our calculations. Specific computational details regarding PCM formalisms are extensively described in related reviews. ${ }^{20,21}$ As a sophisticated solvation model, in addition to the dominant electrostatic contribution to the overall solvent-solute interaction, the extended PCM model also incorporates other contributions, i.e., Pauli repulsion and dispersion, ${ }^{10}$ and takes into account the portion of the solute electric charges lying outside the defined cavity, ${ }^{11,12}$ which may be important in dealing with anions. All these features enable a comprehensive study of solvent-solute reaction field effects upon the (hyper)polarizabilities.

In the present research, $\left[\mathrm{MS}_{4}\right]^{q-}$ and $\left[\mathrm{MO}_{4}\right]^{q-}$ were fixed to their gas phase, tetrahedral geometries optimized at the RHF level using effective core potentials (ECPs) plus valence basis set (VBS) scheme $\operatorname{SBK}(\mathrm{d})$ basis set. ${ }^{22,23}$ They were not reoptimized in solution to facilitate a direct comparison of the results of (hyper)polarizabilities with those obtained from gasphase calculations. Furthermore, previous research suggests that solvent effects on geometry are small for covalently bonded species ${ }^{13}$ and that moderate changes in the geometry of the metal complexes have only a moderate effect on calculated NLO properties. $^{4 \mathrm{a}}$

On the basis of a previous comprehensive study of basis set effects on NLO properties of $\left[\mathrm{MO}_{4}\right]^{q-}$, a the standard SBK$(\mathrm{d})^{22}$ and the augmented SBK $+(\text { ELP }- \text { pd })^{24}$ basis sets were employed for TMs and oxygen, respectively, unless mentioned otherwise. The latter is the $\mathrm{SBK}^{22}$ basis set augmented with a

\begin{tabular}{|c|c|c|c|}
\hline & \multicolumn{3}{|c|}{$\begin{array}{l}\text { exponents of uncontracted } \\
\text { diffuse and polarization } \\
\text { functions for sulfur }\end{array}$} \\
\hline & $\mathrm{s}$ & $\mathrm{p}$ & $\mathrm{d}$ \\
\hline basis $\mathrm{A}\left(\mathrm{SBK}(\mathrm{d})^{22}\right)$ & & & 0.65 \\
\hline basis B & 0.0507 & 0.0405 & 0.0405 \\
\hline basis $\mathrm{C}$ & 0.0507 & 0.0405 & $0.0405,0.1625$ \\
\hline basis $\mathrm{D}$ & 0.0507 & 0.0405 & $0.0405,0.1625,0.65$ \\
\hline basis $\mathrm{E}$ & 0.0507 & 0.0405 & $0.0405,0.1625,0.65,2.6$ \\
\hline
\end{tabular}

TABLE 1: Sulfur Basis Set Augmentation

set of diffuse $\mathrm{s}(\zeta=0.06)$, diffuse $\mathrm{p}(\zeta=0.05)$, and polarization $\mathrm{d}(\zeta=0.9,0.13)$ functions extracted from Dykstra's electrical properties (ELP) basis. ${ }^{25}$ Details about the competence of these basis sets and the ECPs for NLO calculations of inorganic TM complexes can be found elsewhere. ${ }^{4 a}$

For $\left[\mathrm{MS}_{4}\right]^{q-}$, following the previously developed basis set criteria, the SBK(d) set was still employed for TMs. In a fashion similar to the oxygen NLO basis set, an extended basis set for sulfur was constructed and identified to be able to give accurate (hyper)polarizabilities for $\left[\mathrm{MS}_{4}\right]^{q-}$ without compromising computational efficiency.

Initial sulfur basis set tests were carried out on $\mathrm{CS}_{2}$, one of the most frequently used test molecules in both experimental and computational NLO research, and $\left[\mathrm{ReS}_{4}\right]^{-}$. The latter, with the least negative charge and stable in the gas phase, was used as a representative $\left[\mathrm{MS}_{4}\right]^{q-}$, for there is no available neutral species in this series. To augment the standard SBK(d) basis set, diffuse $s, p$, and polarization $d$ functions were mainly extracted from the $6-311++\mathrm{G}(3 \mathrm{df}, 3 \mathrm{pd})$ basis $\left(\xi_{\mathrm{p}}=0.0405\right.$; $\left.\zeta_{\mathrm{d}}=2.6,0.65,0.1625\right),{ }^{26}$ with one additional even-tempered diffuse $d$ function $\left(\xi_{d}=0.0405\right)$. The exponent for the diffuse s function was changed slightly from 0.0405 to 0.0507 as in the aug-cc-pVDZ basis set. ${ }^{27}$ Descriptions for the augmented sulfur basis sets and calculated (hyper)polarizabilities are given in Tables 1 and 2, respectively.

In Table 2, with the increasing size of the augmented sulfur basis set, $\langle\alpha\rangle$ and $\langle\gamma\rangle$ values for $\mathrm{CS}_{2}$ and $\left[\mathrm{ReS}_{4}\right]^{-}$are well converged at both the RHF and MP2 levels. However, there are some slight variations for smaller sets. For example, basis B, Table 1, yields a calculated $\langle\gamma\rangle_{\mathrm{RHF}}$ for $\mathrm{CS}_{2}$ that is larger than that obtained from the most extended basis E. For $\mathrm{CS}_{2}$, the converged $\langle\alpha\rangle$ is comparable with the experimental value, and the converged $\langle\gamma\rangle$ results $(\approx 11000$ au at the RHF level and $\approx 13900$ au at the MP2 level) are in good agreement with those reported with more expensive extended all-electron basis set approaches (11373 $\mathrm{au}^{29}$ and $12801 \mathrm{au}^{30}$ at RHF level and 14270 $\mathrm{au}^{29}$ at MP2 level). Overall, $\langle\alpha\rangle$ and $\langle\gamma\rangle$ results obtained from the second largest set, basis $\mathrm{D}$, met the converged level of $\langle\alpha\rangle$ and $\langle\gamma\rangle$ with very good accuracy (differences of less than $1 \%$ ) and better computational efficiency at both the RHF and MP2 levels of theory. Thus, this sulfur basis set was chosen for further calculations of $\left[\mathrm{MS}_{4}\right]^{q-}$. Basis set $\mathrm{D}$ has one more diffuse $\mathrm{d}$ function in comparison with the SBK $+(\mathrm{ELP}-\mathrm{pd})$ oxygen basis set used previously for $\left[\mathrm{MO}_{4}\right]^{q-}$. This is reasonable since sulfur's electron density distribution is more extended than that of oxygen, and thus more diffuse functions are required to describe NLO properties of species involving sulfur.

Another comment on basis sets is warranted. Basis set effects observed at the RHF level are somewhat different from those with MP2-correlated wave functions. It is seen in Table 2 that electron correlation effects are larger and most likely overestimated for smaller basis sets. For example, for $\left[\mathrm{ReS}_{4}\right]^{-}$, the electron correlation effect using the largest basis sets $\mathrm{D}$ and $\mathrm{E}$ is about $11 \%$ and $33 \%$ for $\langle\alpha\rangle$ and $\langle\gamma\rangle$, respectively. For the 
TABLE 2: Polarizability $(\langle\alpha\rangle)$ and Second Hyperpolarizability $(\langle\gamma\rangle)$ (in au) for $\mathrm{CS}_{2}$ and $\left[\operatorname{ReS}_{4}\right]^{-}$

\begin{tabular}{|c|c|c|c|c|c|c|c|c|}
\hline \multirow[b]{2}{*}{$\mathrm{S}$ basis set } & \multicolumn{4}{|c|}{$\mathrm{CS}_{2}{ }^{a}$} & \multicolumn{4}{|c|}{$\left[\operatorname{ReS}_{4}\right]^{-}$} \\
\hline & $\langle\alpha\rangle_{\mathrm{RHF}}$ & $\langle\alpha\rangle_{\mathrm{MP} 2}$ & $\langle\gamma\rangle_{\mathrm{RHF}}$ & $\langle\gamma\rangle_{\mathrm{MP} 2}$ & $\langle\alpha\rangle_{\mathrm{RHF}}$ & $\langle\alpha\rangle_{\mathrm{MP} 2}$ & $\langle\gamma\rangle_{\mathrm{RHF}}$ & $\langle\gamma\rangle_{\mathrm{MP} 2}$ \\
\hline basis A $(\mathrm{SBK}(\mathrm{d}))$ & 47.2 & 48.9 & 3651 & 4110 & 129.7 & & 3553 & \\
\hline basis B & 47.8 & 52.5 & 12727 & 17763 & 140.3 & 166.7 & 59952 & 89939 \\
\hline basis C & 53.0 & 57.2 & 10094 & 13395 & 148.3 & 170.9 & 62076 & 85681 \\
\hline basis D & 55.0 & 57.0 & 10915 & 13759 & 151.2 & 168.7 & 64567 & 85794 \\
\hline basis $\mathrm{E}$ & 55.4 & 57.2 & 11020 & 13910 & 151.7 & 169.0 & 65069 & 86383 \\
\hline $\exp ^{27}$ & 59.0 & & & & & & & \\
\hline
\end{tabular}

${ }^{a}$ Extended basis set for $\mathrm{C}$ was the $6-31 \mathrm{G}(3 \mathrm{~d})+$ pd set. ${ }^{29}$ Experimental geometry was used for $\mathrm{CS}_{2}(\mathrm{C}-\mathrm{S}=1.5526 \AA) .{ }^{28}$

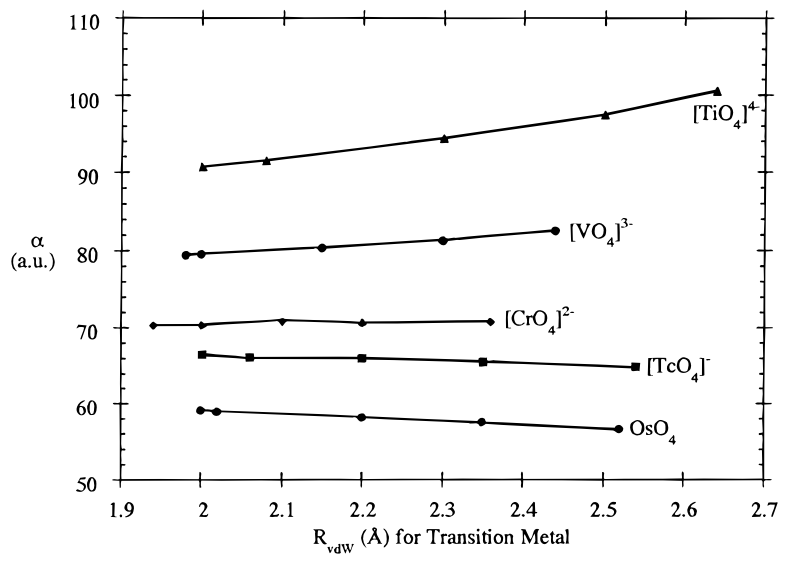

Figure 1. Dependence of polarizabilities on van der Waals radii of TMs.

smaller basis B, the correlation effect is about $19 \%$ for $\langle\alpha\rangle$ and $50 \%$ for $\langle\gamma\rangle$, resulting in even larger $\langle\alpha\rangle_{\mathrm{MP} 2}$ and $\langle\gamma\rangle_{\mathrm{MP} 2}$ values than those obtained from the largest basis sets. In the reported calculations, the same basis set (i.e., basis D) was used with RHF and MP2 wave functions for a more accurate assessment of the NLO response to chemical and computational factors.

\section{Results and Discussions}

Effect of Transition Metal van der Waals Radii on Solute (Hyper)Polarizabilities. As an advanced solvation model, PCM has as one of its values a sophisticated treatment of the cavity size. To do so, the formalism requires the van der Waals radii $\left(R_{\mathrm{vdW}}\right)$ of the atoms of the solute molecule. Thus, the first practical issue for a study of TM complexes is how to define the $R_{\mathrm{vdW}}$ for TMs since there are little available experimental or calculated data.

In practice, there are several different ways to estimate $R_{\mathrm{vdW}}$. Thus, first, the dependence of the calculated values of (hyper)polarizabilities on variations in the cavity size for some representative $\left[\mathrm{MO}_{4}\right]^{q-}$ from different TM triads was assessed. The cavity size was varied by varying $R_{\mathrm{vdW}}$ of the TMs. The calculations were done for water solvent and with the SBK + (ELP - pdd') basis set for oxygen. ${ }^{31}$ The results for $\langle\alpha\rangle_{\mathrm{PCM}}$ and $\langle\gamma\rangle_{\mathrm{PCM}}$ are given in Figures 1 and 2, respectively. In the two figures, the minima of the estimated $R_{\mathrm{vdW}}$ for TMs were either $2.0 \AA$ or those obtained from Bondi's approximation using the corresponding covalent radii $\left(R_{\text {covalent }}\right)\left(R_{\mathrm{vdW}}(\AA)=R_{\text {covalent }}\right.$ $(\AA)+0.76(\AA)),{ }^{32}$ whichever is smaller. The upper limits for the estimated $R_{\mathrm{vdW}}$ were equal to two times the corresponding $R_{\text {covalent. Overall, the results show moderate and near-parallel }}$ variations in calculated values of $\langle\alpha\rangle_{\mathrm{PCM}}$ and $\langle\gamma\rangle_{\mathrm{PCM}}$ within a very large range of estimated $R_{\mathrm{vdW}}$ for the test species. This suggests a relatively small effect on (hyper)polarizabilities within PCM from varying the $R_{\mathrm{vdW}}$ of the TMs. Thus, in the remaining calculations, the $R_{\mathrm{vdW}}$ was set to $2.0 \AA$ for all TMs to facilitate comparisons among different complexes.

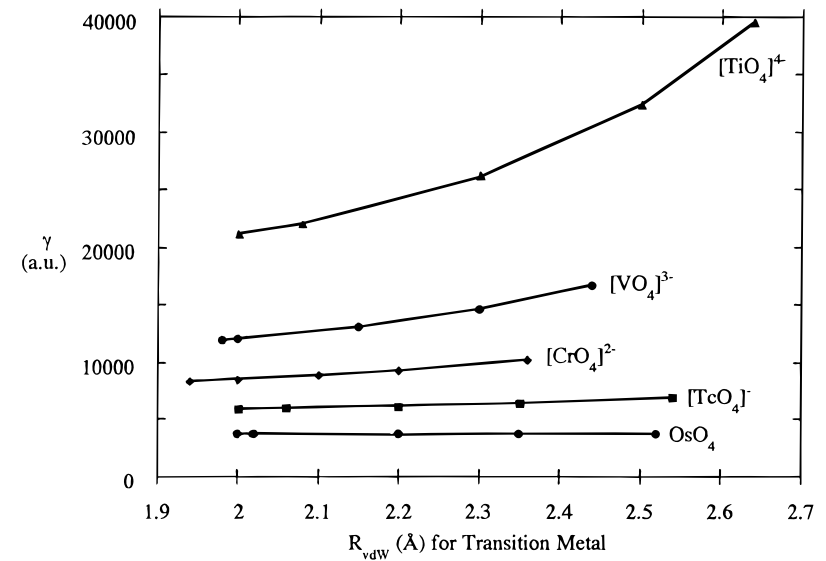

Figure 2. Dependence of second hyperpolarizabilities on van der Waals radii of TMs.

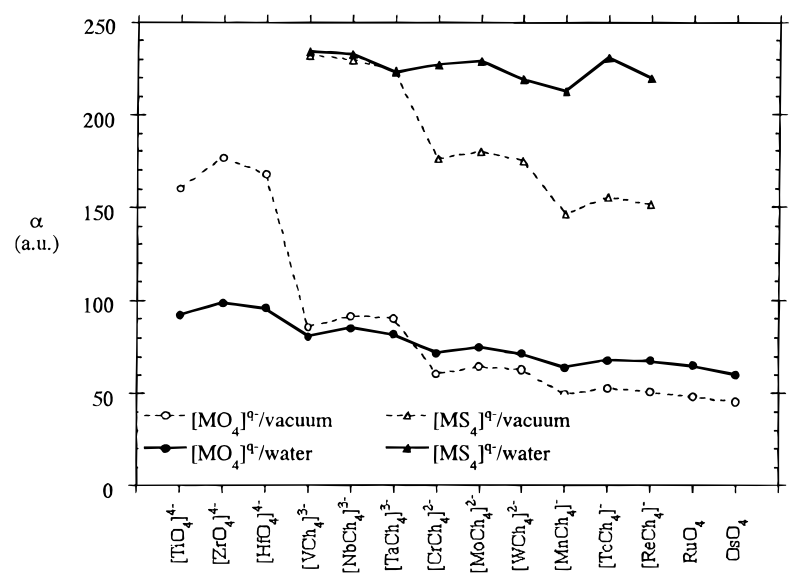

Figure 3. Polarizabilities of TM metalates and thiometalates, $\left[\mathrm{MCh}_{4}\right]^{q-}$ $(\mathrm{Ch}=\mathrm{O}, \mathrm{S})$, in solution.

Solvent Effects on (Hyper)Polarizabilities of TM (Thio)Metalates. To systematically study solvent effects on NLO properties of TM complexes, (hyper)polarizabilities in water solvent were calculated for the series of $\left[\mathrm{MO}_{4}\right]^{q-}$ and $\left[\mathrm{MS}_{4}\right]^{q-}$. The results for polarizabilities $\left(\langle\alpha\rangle_{\mathrm{PCM}}\right)$ and second hyperpolarizabilities $\left(\langle\gamma\rangle_{\mathrm{PCM}}\right)$ are given in Figures 3 and 4, respectively. The corresponding results from the gas-phase calculations, $\langle\alpha\rangle_{\text {vacuum }}$ and $\langle\gamma\rangle_{\text {vacuum, }}$ are also given in the same figures as references.

Champagne et al. reported that upon solvation, there is a decrease in the excitation energy of the singly exited, dipoleallowed electronic state. ${ }^{13}$ As this is the most important contribution to polarization, the effect is to increase (hyper)polarizabilities of the solute over those obtained in a vacuum. This has been shown in several different works. ${ }^{13-16}$ For the present results, Figures 3 and 4, positive solvent effects are seen for $\langle\alpha\rangle_{\mathrm{PCM}}$ for neutral and less negatively charged $\left[\mathrm{MO}_{4}\right]^{q-}$ and $\left[\mathrm{MS}_{4}\right]^{q-}$ (i.e., (thio)metalates with TMs in groups 8, 7, and 6, 


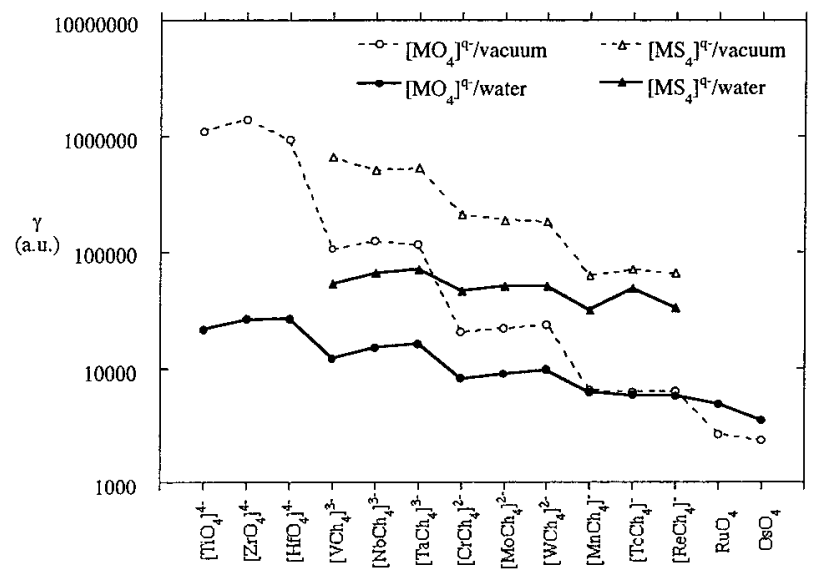

Figure 4. Second hyperpolarizabilities of TM metalates and thiometalates, $\left[\mathrm{MCh}_{4}\right]^{q-}(\mathrm{Ch}=\mathrm{O}, \mathrm{S})$, in solution.

respectively) and for $\langle\gamma\rangle_{\mathrm{PCM}}$ for neutral $\left[\mathrm{MO}_{4}\right]^{q-}$ (i.e., $\mathrm{RuO}_{4}$ and $\left.\mathrm{OsO}_{4}\right)$. The abnormally larger $\langle\alpha\rangle_{\text {vacuum }}$ and $\langle\gamma\rangle_{\text {vacuum }}$ versus $\langle\alpha\rangle_{\mathrm{PCM}}$ and $\langle\gamma\rangle_{\mathrm{PCM}}$, respectively, for the other species are a consequence of the magnified results obtained from calculations of unbound anions in a vacuum. The extremely large differences in $\langle\alpha\rangle_{\mathrm{PCM}}$ and $\langle\gamma\rangle_{\mathrm{PCM}}$ seen for some species, Figures 3 and 4 , suggest strongly that linear polarizabilities of highly negatively charged $(-q>2)$ inorganic TM (thio)metalates and second hyperpolarizabilities of all anionic inorganic TM (thio)metalates cannot be reliably modeled in the gas phase due to the unsuitability of Hartree-Fock wave functions for describing highly anionic species in the gas phase. Calculations of these species in a vacuum give rise to a systematic overestimation of NLO properties. Overall, such overestimation and magnification are more dramatic for more negatively charged species and much more serious for $\langle\gamma\rangle_{\text {vacuum }}$ than for $\langle\alpha\rangle_{\text {vacuum. }}$. From this point of view, modeling NLO properties in solution is not only important for assessing solvent effects upon NLO properties of interest, but it also provides the only reliable way to model and predict NLO properties for a series of anionic inorganic complexes.

There are two more points of interest in Figures 3 and 4 regarding solvent effects on (hyper)polarizabilities:

(1) For species with a negative charge less than 3, nearparallel trends in $\langle\alpha\rangle_{\mathrm{PCM}}$ and $\langle\alpha\rangle_{\text {vacuum }}$ are seen for both the $\left[\mathrm{MO}_{4}\right]^{q-}$ and $\left[\mathrm{MS}_{4}\right]^{q-}$ series. $\langle\alpha\rangle_{\mathrm{PCM}}$ averages $\approx 37 \%$ and $\approx 26 \%$ above $\langle\alpha\rangle_{\text {vacuum }}$ for $\left[\mathrm{MS}_{4}\right]^{q-}$ and $\left[\mathrm{MO}_{4}\right]^{q-}$, respectively. Therefore, solvent effects on linear polarizabilities are stronger for $\left[\mathrm{MS}_{4}\right]^{q-}$ than for $\left[\mathrm{MO}_{4}\right]^{q-}$. Solvent effects on second hyperpolarizabilities can only be reliably assessed from the two neutral TM oxides, $\mathrm{RuO}_{4}$ and $\mathrm{OsO}_{4}$. For these two species, much larger solvent effects are seen on $\langle\gamma\rangle$ than $\langle\alpha\rangle$. The increase in $\langle\gamma\rangle$ is $83 \%$ and $51 \%$ for $\mathrm{RuO}_{4}$ and $\mathrm{OsO}_{4}$, respectively, while the corresponding increase in their $\langle\alpha\rangle$ values is $35 \%$ and $32 \%$, respectively.

(2) For species with a negative charge less than 3 , while there are little differences in solvent effects on $\langle\alpha\rangle$ for species within the same TM triad, the results for $\mathrm{RuO}_{4}$ and $\mathrm{OsO}_{4}$ seem to indicate a clearly weaker solvent effect on $\langle\gamma\rangle$ for the $\left[\mathrm{MO}_{4}\right]^{q-}$ in the third transition series as compared to their congeners in lower series.

Trends in (Hyper)Polarizabilities in Solution as a Function of TM. Reliable calculations with the PCM solvation model enable a systematic study of the important trends in $\langle\alpha\rangle$ and $\langle\gamma\rangle$ for $\left[\mathrm{MS}_{4}\right]^{q-}$ and $\left[\mathrm{MO}_{4}\right]^{q-}$ as a function of the TM. Figure 3 shows that, within each TM triad of (thio)metalates, trends observed in $\langle\alpha\rangle_{\mathrm{PCM}}$ and $\langle\gamma\rangle_{\mathrm{PCM}}$ are almost parallel to and thus follow the corresponding trends in $\langle\alpha\rangle_{\text {vacuum }}$ and $\langle\gamma\rangle_{\text {vacuum, }}$,

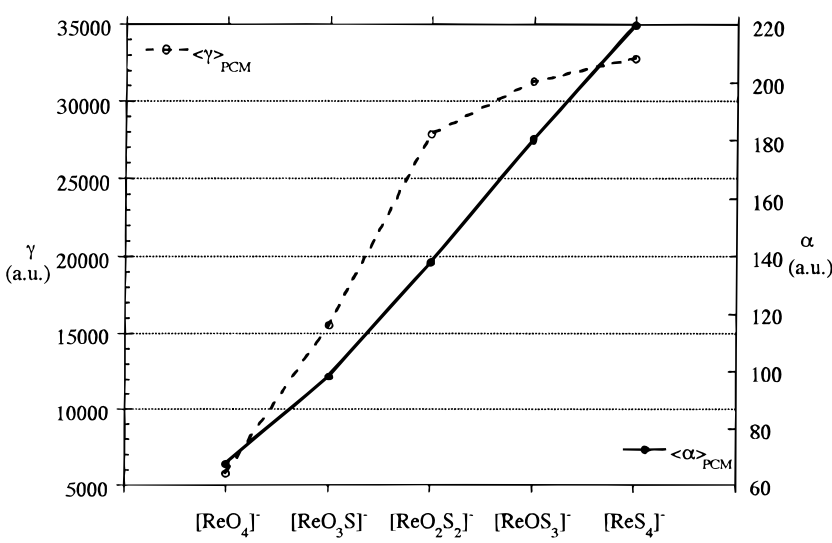

Figure 5. Polarizabilities and second hyperpolarizabilities of (thio)rhenate in solution.

respectively. Generally, variations in $\langle\alpha\rangle_{\mathrm{PCM}}$ values are quite small within the same TM triads for both the $\left[\mathrm{MO}_{4}\right]^{q^{-}}$and $\left[\mathrm{MS}_{4}\right]^{q-}$ series, with the species in the second transition series having a slightly bigger calculated $\langle\alpha\rangle_{\mathrm{PCM}}$ in most cases. Overall, there is a very gentle decrease in $\langle\alpha\rangle_{\mathrm{PCM}}$ from group 4 to group 8 across the periodic table. The ratios between the biggest and the smallest $\langle\alpha\rangle_{\mathrm{PCM}}$ values are $\approx 1.6\left(\left[\mathrm{ZrO}_{4}\right]^{4-} / \mathrm{OsO}_{4}\right)$ and $\approx 1.1$ $\left(\left[\mathrm{VS}_{4}\right]^{3-} /\left[\mathrm{MnS}_{4}\right]^{-}\right)$for the $\left[\mathrm{MO}_{4}\right]^{q-}$ and $\left[\mathrm{MS}_{4}\right]^{q-}$ series, respectively. The range of $\langle\alpha\rangle_{\mathrm{PCM}}$ is $\approx 39$ au and $\approx 21$ au for the $\left[\mathrm{MO}_{4}\right]^{q-}$ and $\left[\mathrm{MS}_{4}\right]^{q-}$ series, respectively.

Trends in $\langle\gamma\rangle_{\mathrm{PCM}}$ values, Figure 4, generally follow those observed from $\langle\alpha\rangle_{\mathrm{PCM}}$, Figure 3. However, unlike $\langle\alpha\rangle_{\mathrm{PCM}}$, in most cases the third transition series congeners possess a slightly bigger calculated $\langle\gamma\rangle_{\mathrm{PCM}}$ for (thio)metalates within a TM triad. For $\langle\gamma\rangle_{\mathrm{PCM}}$, the decrease from left to right across the periodic table is larger than for $\langle\alpha\rangle_{\mathrm{PCM}}$. The ratios between the biggest and the smallest $\langle\gamma\rangle_{\mathrm{PCM}}$ values are $\approx 7.5\left(\left[\mathrm{HfO}_{4}\right]^{4-} / \mathrm{OsO}_{4}\right)$ and $\approx 2.3\left(\left[\mathrm{TaS}_{4}\right]^{3-} /\left[\mathrm{MnS}_{4}\right]^{-}\right)$for each series. The range of $\langle\gamma\rangle_{\mathrm{PCM}}$ is $\approx 23000$ au and $\approx 39000$ au for the $\left[\mathrm{MO}_{4}\right]^{q-}$ and $\left[\mathrm{MS}_{4}\right]^{q^{-}}$ series, respectively.

Trends in (Hyper)Polarizabilities as a Function of Chalcogen. Reliable calculations with the PCM solvation model also enable a study of another important effect on modeling NLO properties of inorganic TM complexes, the ligand modification effect. The near-parallel trends in both $\langle\alpha\rangle_{\mathrm{PCM}}$ and $\langle\gamma\rangle_{\mathrm{PCM}}$ of $\left[\mathrm{MS}_{4}\right]^{q-}$ and $\left[\mathrm{MO}_{4}\right]^{q-}$ series, Figures 3 and 4 , respectively, give rise to an average 3.1 times larger $\langle\alpha\rangle_{\mathrm{PCM}}$ and 5.4 times larger $\langle\gamma\rangle_{\mathrm{PCM}}$ when a $\left[\mathrm{MO}_{4}\right]^{q-}$ is replaced with a $\left[\mathrm{MS}_{4}\right]^{q-}$ congener. The corresponding average enhancement caused by one oxoto-sulfido substitution is $\approx 52 \%$ in $\langle\alpha\rangle(38 \mathrm{au})$ and $\approx 110 \%$ in $\langle\gamma\rangle(10000 \mathrm{au})$.

To further demonstrate the ligand modification effects by changing oxo with sulfido groups more quantitatively, a series of anionic (thio)rhenate $\left(\left[\mathrm{ReO}_{x} \mathrm{~S}_{4-x}\right]^{-}(x=4-0)\right)$ was calculated with PCM in water, with the initial four oxo groups replaced by four sulfido groups in four substitutions. Results of $\langle\alpha\rangle$ and $\langle\gamma\rangle$ values are shown in Figure 5.

Replacing a $\mathrm{Re}=\mathrm{O}$ linkage with a $\mathrm{Re}=\mathrm{S}$ linkage causes a large and linear increase in $\langle\alpha\rangle_{\mathrm{PCM}}$, Figure 5. One oxo-to-sulfido substitution leads to an average enhancement of $56 \%(\approx 38 \mathrm{au})$ in $\langle\alpha\rangle_{\mathrm{PCM}}$. The first two oxo-to-sulfido substitutions lead to nearlinear and much larger increases in $\langle\gamma\rangle_{\mathrm{PCM}}$, with one substitution resulting in an average enhancement of $190 \%(\approx 11000 \mathrm{au})$. It is very interesting to find that this result compares well with the 11900 and 11060 au enhancement in experimental $\gamma$ (measured at $790 \mathrm{~nm}$ ) in tetrahydrofuran-to-tetrahydrothiophene and furan-to-thiophene substitutions, respectively, reported by Kamada et al. ${ }^{6}$ This might suggest a rough level of contributions 


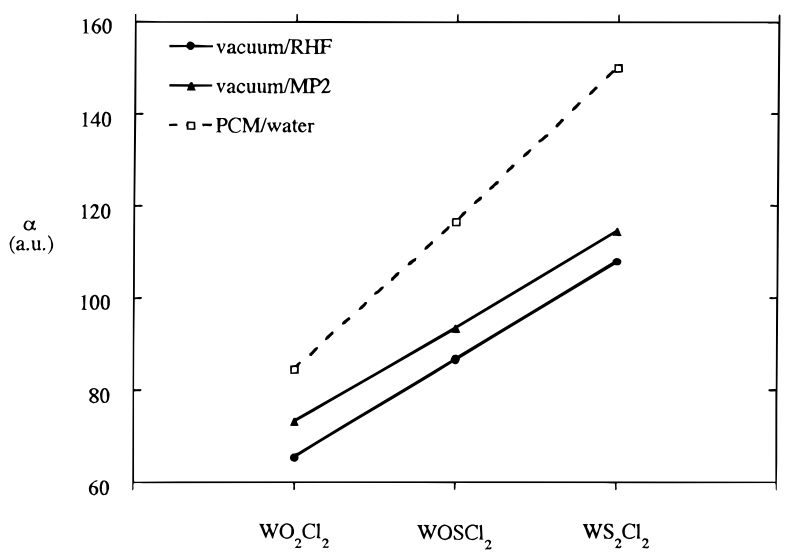

Figure 6. Polarizabilities of $\mathrm{WO}_{x} \mathrm{~S}_{2-x} \mathrm{Cl}_{2}(x=2-0)$.

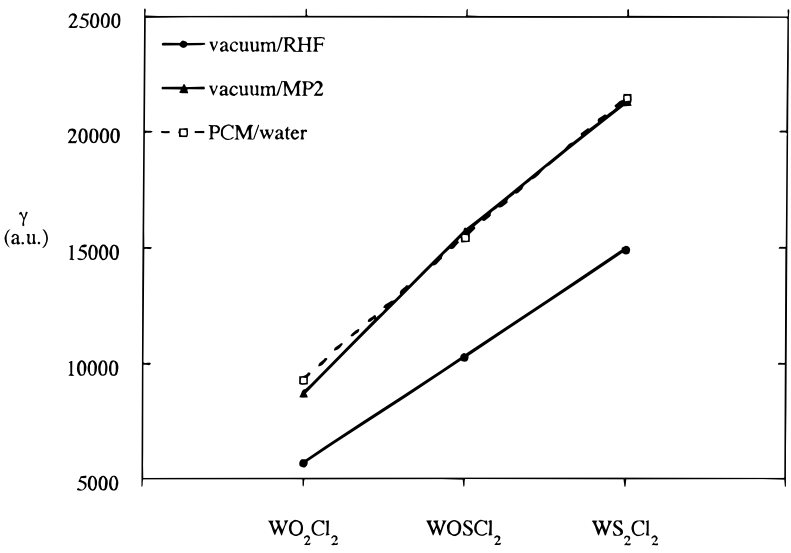

Figure 7. Second hyperpolarizabilities of $\mathrm{WO}_{x} \mathrm{~S}_{2-x} \mathrm{Cl}_{2}(x=2-0)$.

of sulfur to the $\gamma$ response. However, in Figure 5, the enhancement in $\langle\gamma\rangle_{\mathrm{PCM}}$ caused by the last two oxo-to-sulfido substitutions becomes much lower and seems to saturate. As an overall average, from $\left[\mathrm{ReO}_{4}\right]^{-}$to $\left[\mathrm{ReS}_{4}\right]^{-}$, one oxo-to-sulfido substitution increases $\langle\gamma\rangle$ by $117 \%$ (6740 au).

Comparison of Solvent Effect and Electron Correlation Effect. The current PCM solvation model does not include electron correlation effects. However, it is of interest to compare the solvent effect at the RHF level with the electron correlation effect on (hyper)polarizabilities. For this purpose, neutral tungstenyl complexes $\left(\mathrm{WO}_{x} \mathrm{~S}_{2-x} \mathrm{Cl}_{2}(x=2-0)\right)$ were calculated in the gas phase at both the RHF and the MP2 levels and with PCM in water. The most extended basis sets developed, the $\mathrm{SBK}+\mathrm{ELP}^{33}$ and basis E, were used for $\mathrm{O}$ and $\mathrm{S}$, respectively. The basis set for $\mathrm{Cl}$ was also a very extended one: $\mathrm{SBK}(\mathrm{d})$ augmented by one $\mathrm{s}$, two $\mathrm{p}$, and two d diffuse and polarization functions $\left(\xi_{\mathrm{s}}=0.0483 ;{ }^{34} \xi_{\mathrm{p}}=0.0483,{ }^{34} 0.0037 ;{ }^{35} \xi_{\mathrm{d}}=0.1875,{ }^{34}\right.$ $\left.0.046875^{36}\right)$. Results for $\langle\alpha\rangle$ and $\langle\gamma\rangle$ are shown in Figures 6 and 7 , respectively.

For the three complexes calculated, solvent effects at the RHF level yield an average increase in $\langle\alpha\rangle$ of $34 \%$ and in $\langle\gamma\rangle$ of $50 \%$. In comparison with the solvent effect, the average MP2 correlation effect of $9 \%$ on $\langle\alpha\rangle$ in the gas phase is much less dramatic (Figure 6). However, the average 50\% MP2 correlation effect on $\langle\gamma\rangle$ in the gas phase is about the same size and importance as the solvent effect (Figure 7). This suggests that there can be a remarkable correlation effect to give rise to a significant increase in the calculated second hyperpolarizabilities in solution. However, correlation effects assessed in the gasphase calculations may not be directly extended to solution.

Extended PCM Calculations of (Hyper)Polarizabilities in Solution. The electrostatic effect is the dominant one in
TABLE 3: Polarizabilities $(\langle\alpha\rangle)$ and Second Hyperpolarizabilities $(\langle\gamma\rangle)$ (in au) of $\mathrm{OsO}_{4}$ and $\left[\mathrm{TiO}_{4}\right]^{4-}$ in Vacuum and in Water by Extended PCM Calculations

\begin{tabular}{lccrrr}
\hline \multirow{2}{*}{ method } & \multicolumn{2}{c}{$\mathrm{OsO}_{4}$} & & \multicolumn{2}{c}{$\left[\mathrm{TiO}_{4}\right]^{4-}$} \\
\cline { 2 - 3 } & $\langle\alpha\rangle$ & $\langle\gamma\rangle$ & & \multicolumn{1}{c}{$\langle\alpha\rangle$} & \multicolumn{1}{c}{$\langle\gamma\rangle$} \\
\hline vacuum/RHF & 44.8 & 2394 & & 156.2 & 968263 \\
PCM (el) (standard PCM) & 59.0 & 3621 & & 90.6 & 20672 \\
PCM (+q) & 59.7 & 3731 & & 96.1 & 23512 \\
PCM (+r) & 58.4 & 3100 & & 87.1 & 16849 \\
PCM (+d) & 59.1 & 3602 & & 90.7 & 20705 \\
PCM (+r+d) & 58.4 & 3083 & & 87.1 & 16863 \\
PCM (+q+r) & 59.0 & 3192 & & 91.9 & 19049 \\
PCM (+q+d) & 59.7 & 3730 & & 96.1 & 23824 \\
PCM (+q+r+d) & 59.0 & 3190 & &
\end{tabular}

solvent-solute interactions and thus should be the most important factor in the study of solvent effects on (hyper)polarizabilities. However, there are also other contributing factors to solvent-solute interactions. These include Pauli repulsion and dispersion ${ }^{10}$ as well as the portion of the solute electric charges lying outside the defined cavity. ${ }^{11,12}$ These may therefore also have some effects on solute (hyper)polarizabilities. Some computations reported recently on prototypical organic molecules suggested that these contributions generally have only small effects, and for most cases, they cancel each other and thus are negligible. ${ }^{13,14}$ In the present research, we have until now employed the standard PCM solvation model considering only the electrostatic solvent-solute interactions in the calculations of inorganic TM complexes. Here, we now employ the extended PCM solvation model on two representative species, the neutral $\mathrm{OsO}_{4}$ and the most anionic $\left[\mathrm{TiO}_{4}\right],{ }^{4-}$ to assess different solvent effects on (hyper)polarizabilities. The smaller SBK $+\left(\text { ELP }- \text { pdd }^{\prime}\right)^{31}$ oxygen basis was used in the calculations. Results are organized in Table 3. Results from the gas phase at the RHF level are also listed as a reference. Different PCM methods refer to the calculations in which different solvent-solute interactions were taken into account. They are respectively electrostatic (el, i.e., the standard PCM solvation model), electrostatic and solute electrons outside the cavity $(+q)$, electrostatic and Pauli repulsion $(+r)$, electrostatic and dispersion $(+\mathrm{d})$, and electrostatic and a combination of two or three of these other contributions (i.e., $+\mathrm{r}+\mathrm{d},+\mathrm{q}+\mathrm{r},+\mathrm{q}+\mathrm{d}$, and $+q+r+d)$.

For the neutral $\mathrm{OsO}_{4}$, the calculated charge outside the cavity is 0.1 electrons. The corresponding correction has a small positive effect, resulting in about a $1 \%$ increase in $\langle\alpha\rangle$ and a $3 \%$ increase in $\langle\gamma\rangle$. Pauli repulsion, with its resistant effect on the extension of the solute charge distribution, has a small negative effect on $\langle\alpha\rangle$ (1\% decrease) and a bigger negative effect on $\langle\gamma\rangle$ (14\% decrease). The effect of dispersion on $\langle\alpha\rangle$ and $\langle\gamma\rangle$ is very small $(<1 \%)$. Overall, corrections from employing the extended PCM solvation model are found to be negligible for $\langle\alpha\rangle$ and to decrease $\langle\gamma\rangle$ by about $12 \%$.

For $\left[\mathrm{TiO}_{4}\right],{ }^{4-}$ the calculated charge outside the cavity is 0.7 electrons, much more than the neutral $\mathrm{OsO}_{4}$. Thus, this correction has a bigger positive effect, resulting in a $6 \%$ increase in $\langle\alpha\rangle$ and a $14 \%$ increase in $\langle\gamma\rangle$. The negative effect caused by Pauli repulsion is also stronger for $\left[\mathrm{TiO}_{4}\right]^{4-}$ due to the more extended distribution of its highly negative charge; $\langle\alpha\rangle$ decreases by $6 \%$, while $\langle\gamma\rangle$ decreases by $19 \%$. As in the above case for $\mathrm{OsO}_{4}$, the effect of dispersion on $\langle\alpha\rangle$ and $\langle\gamma\rangle$ is also very small $(<1 \%)$. Overall, corrections from employing the extended PCM solvation model are found to be smaller for $\left[\mathrm{TiO}_{4}\right]^{4-}$ than for $\mathrm{OsO}_{4}$. They increase $\langle\alpha\rangle$ by $2 \%$ and decrease $\langle\gamma\rangle$ by $5 \%$.

In conclusion, these results verified that subordinate effects other than the dominant electrostatic solvent-solute interactions 
are small for $\langle\alpha\rangle$ and $\langle\gamma\rangle$. The two relatively larger effects caused by the Pauli repulsion and charge escaping outside the cavity are different in sign and tend to partly cancel each other, while dispersion effects are minimal. The results also show that these subordinate effects are nearly additive. Thus, the overall effect can be estimated from the addition of each individual effect. Overall, these additional factors have negligible impact on $\langle\alpha\rangle$. They do decrease $\langle\gamma\rangle$ by about $10 \%$. However, the effect is small in comparison with the much bigger changes induced by electrostatic interactions.

\section{Conclusions}

A computational investigation of the polarizabilities and the second hyperpolarizabilities of inorganic TM thiometalates and metalates in solution is reported. Several important results have been reached, the most important of which are summarized below.

(1) A computationally accurate, yet efficient, extended ECP sulfur basis set was identified for calculations of NLO properties of inorganic TM thiometalates. In comparison with the extended oxygen basis set for NLO calculations of inorganic TM metalates developed previously, it is found that the sulfur basis set requires an additional diffuse d function to yield wellconverged results for (hyper)polarizabilities. It is also found that correlation effects tend to be magnified with smaller sulfur basis sets.

(2) It is found that the PCM solvation model provides a reliable way to model NLO properties of anionic inorganic complexes when these cannot be reliably modeled in the gas phase. Thus, NLO properties of a series of negatively charged inorganic complexes can be predicted upon solvation. This may prove to be important for the design of novel inorganic materials with large NLO potentials.

(3) Overall, subordinate contributions to solute-solvent interactions have negligible effects on (hyper)polarizabilities of inorganic TM complexes in solution.

(4) Solvent effects, which mainly correspond to the electrostatic solute-solvent interactions, increase $\langle\alpha\rangle$ and $\langle\gamma\rangle$ of inorganic TM complexes. The effects are much larger for $\langle\gamma\rangle$ than for $\langle\alpha\rangle$ and are larger for TM thiometalates than for metalates. For $\langle\alpha\rangle$, solvent effects are found to be more important than electron correlation effects in the effort to bridge the gap between the computational and experimental results. For $\langle\gamma\rangle$, solvent effects and correlation effects are similar in size and importance. This suggests the necessity to take into account correlation effects in modeling hyperpolarizabilities in solution. However, this is prohibited by the current PCM solvation model.

(5) For the series of TM (thio)metalates, the differences in $\langle\alpha\rangle$ and $\langle\gamma\rangle$ within the same TM triad are very small. Across the TM triads, $\langle\alpha\rangle$ and $\langle\gamma\rangle$ decrease from group 4 to group 8 . The decrease in $\langle\alpha\rangle$ is $60 \%$ for metalate series, larger than the $10 \%$ calculated for thiometalate series. For $\langle\gamma\rangle$, this decrease is much more remarkable. It is $650 \%$ for the metalate series and $130 \%$ for the thiometalate series. This suggests that the $\gamma$ response is more sensitive to changing TMs, and a significant enhancement in the $\gamma$ response of inorganic TM complexes can be achieved by using TMs in earlier triads.

(6) The NLO properties of inorganic TM complexes can be significantly enhanced by changing ligand atoms with heavier congeners. The average enhancement caused by one oxo-tosulfido substitution is found to be $52 \%(\approx 38 \mathrm{au})$ and $110 \%$ $(\approx 10000 \mathrm{au})$ for $\langle\alpha\rangle$ and $\langle\gamma\rangle$, respectively.
This research applied a sophisticated solvation model as an effort toward quantitatively modeling and predicting NLO properties of inorganic complexes in a more realistic environment. Because of the lack of experimental data as well as limitations in some computational factors, such as local field effects and electron correlation effects, the present results are hard to compare with experimental results. However, the research identified successful strategies to model the NLO properties of anionic inorganic complexes when they cannot be reliably modeled in the gas phase. Furthermore, on the basis of the results obtained, important suggestions have been forwarded for the practical purpose of predicting nonlinearity and designing novel inorganic NLO materials.

Acknowledgment. T.R.C. acknowledges support of this research through Grant CHE-9614346 from the National Science Foundation (NSF). This work was generously supported by National Computational Science Alliance (NCSA) under Grant CHE970022N and utilized the HP/Convex Exemplar SPP-2000 at National Center for Supercomputing Applications (NCSA). Additionally, H.A.K. and T.R.C. acknowledge the generous support of the NSF for computational chemistry research at the University of Memphis through Grants STI-9602656 (from the Academic Research Infrastructure program) and CHE-9708517 (from the Chemical Research Instrumentation and Facilities program). T.Z. acknowledges a travel scholarship from the Physical Division of the American Chemical Society (ACS) for presenting part of this research at the Symposium in Theory and Modeling of Electronic and NLO Materials at the 218th ACS National Meeting.

\section{References and Notes}

(1) E-mail: tcundari@memphis.edu.

(2) See review books and papers and the related references therein, e.g., (a) Karna, S. P., Yeates, A. T., Eds.; ACS Symposium Series 628; American Chemical Society: Washington, DC, 1996. (b) Kurtz, H. A.; Dudis, D. S. In Reviews in Computational Chemistry; Boyd, D. B., Lipkowitz, K. B., Eds.; Wiley-VCH: New York, 1998; Vol. 12, p 241. (c) Ratner, M. Int. J. Quantum Chem. 1992, 43, 5. (d) Brédas, J. L.; Adant C.; Tackx P.; Persoons, A.; Pierce, B. M. Chem. Rev. 1994, 94, 243. (e) Kanis, D. R.; Ratner, M. A.; Marks, T. J. Chem. Rev. 1994, 94, 195. (f) Stucky, G. D.; Marder, S. R.; Sohn, J. E. In Materials for Nonlinear OpticsChemical Perspectives; Marder, S. R., Sohn, J. E., Stucky, G. D., Eds.; ACS Symposium Series 455; American Chemical Society: Washington, DC, 1991; p 2 (g) Whittall, I. R.; McDonagh, A. M.; Humphrey, M. G.; Samoc, M. Adv. Organomet. Chem. 1998, 42, 291. (h) Whittall, I. R.; McDonagh, A. M.; Humphrey, M. G.; Samoc, M. Adv. Organomet. Chem. 1999, 43, 349.

(3) (a) Munowitz, M.; Jarman, R. H.; Harrison, J. F. Chem. Mater. 1992, 4, 1296. (b) Munowitz, M.; Jarman, R. H.; Harrison, J. F. Chem. Mater. 1993, 5, 661. (c) Munowitz, M.; Jarman, R. H.; Harrison, J. F. Chem. Mater. 1993, 5, 1257.

(4) (a) Cundari, T. R.; Kurtz, H. A.; Zhou, T. J. Phys. Chem. A. 1998, 102, 2962. (b) Cundari, T. R.; Kurtz, H. A.; Zhou, T. Chem. Phys. 1999 $240,205$.

(5) Singh, S. In Handbook of Laser Science and Technology; Weber, M. J., Ed.; CRC Press: Bota Raton, FL, 1986; Vol. 3, p 3.

(6) Kamada, K.; Ueda, M.; Sakaguchi, T.; Ohta, K.; Fukumi, T. Chem. Phys. Lett. 1996, 263, 215; 1997, 267, 402 (erratum).

(7) Rustagi, K. C.; Ducuing, J. Opt. Commun. 1974, 10, 258

(8) Miertus, S.; Scrocco, E.; Tomasi, J. Chem. Phys. 1981, 55, 117.

(9) Cammi, R.; Tomasi, J. J. Comput. Chem. 1995, 16, 1449.

(10) Amovilli, C.; Mennucci, B. J. Phys. Chem. B 1997, 101, 1051.

(11) Mennucci, B.; Tomasi, J. J. Chem. Phys. 1997, 106, 5151.

(12) Chipman, D. M. J. Chem. Phys. 1997, 106, 10194.

(13) Champagne, B.; Mennucci, B.; Cossi, M.; Cammi, R.; Tomasi, J. Chem. Phys. 1998, 238, 153.

(14) Mennucci, B.; Amovilli, C.; Tomasi, J. Chem. Phys. Lett. 1998, 286, 221.

(15) Cammi, R.; Cossi, M.; Tomasi, J. J. Chem. Phys. 1996, 104, 4611.

(16) Cammi, R.; Cossi, M.; Mennucci, B.; Tomasi, J. J. Chem. Phys. 1996, 105, 10556

(17) (a) Karna, S. P.; Dupuis, M. J. Comput. Chem. 1991, 12, 487. (b) Sekino, H.; Bartlett, R. J. J. Chem. Phys. 1986, 85, 976. 
(18) Kurtz, H. A.; Stewart, J. J. P.; Dieter, K. M. J. Comput. Chem. $1990,11,82$.

(19) Schmidt, M. W.; Baldridge, K. K.; Boatz, J. A.; Jensen, J. H.; Koseki, S.; Matsunaga, N.; Gordon, M. S.; Nguyen, K. A.; Su, S.; Windus, T. L.; Elbert, S. T.; Montgomery, J.; Dupuis, M. J. Comput. Chem. 1993, 14, 1347.

(20) Tomasi, J.; Persico, M. Chem. Rev. 1994, 94, 2027

(21) Amovilli, C.; Barone, V.; Cammi, R.; Cancès, E.; Cossi, M.; Mennucci, B.; Pomelli, C. S.; Tomasi, J. Adv. Quantum Chem. 1999, 32 , 227.

(22) Krauss, M.; Stevens, W. J.; Basch, H.; Jasien, P. G. Can. J. Chem. 1992, 70, 612 .

(23) Benson, M. T.; Cundari, T. R.; Lim, S. J.; Nguyen, H. D.; PierceBeaver, K. J. Am. Chem. Soc. 1994, 116, 3955.

(24) Referred to as SBK(d) + Dyk - pd in ref 4a.

(25) Dykstra, C. E.; Liu, S.-Y.; Malik, D. J. Adv. Chem. Phys. 1989, 75,37 .
(26) McLean, A. D.; Chandler, G. S. J. Chem. Phys. 1980, 72, 5639.

(27) Woon, D. E.; Dunning, T. H., Jr. J. Chem. Phys. 1993, 98, 1358.

(28) Handbook of Chemistry and Physics, 78th ed.; CRC Press: Boca Raton, FL, 1997-1998.

(29) Ohta, K.; Sakaguchi, T.; Kamada, K.; Fukumi, T. Chem. Phys. Lett. 1997, 274, 306.

(30) Hamada, T. Chem. Phys. 1996, 211, 171.

(31) Referred to as SBK(d) + Dyk - pdd' in ref 4a.

(32) Bondi, A. J. Phys. Chem. 1964, 68, 441.

(33) Referred to as $\operatorname{SBK}(d)+$ Dyk in ref $4 a$.

(34) Francl, M. M.; Petro, W. J.; Hehre, W. J.; Binkley, J. S.; Gordon, M. S.; DeFrees, D. J.; Pople, J. A. J. Chem. Phys. 1982, 77, 3654.

(35) Dunning, T. H., Jr. J. Chem. Phys. 1970, 53, 2823.

(36) Even-tempered from the former diffuse $d$ function $(\xi=0.1875)$. 